\title{
Phenotypic and Genomic Analyses of Human Strains Belonging or Related to Bifidobacterium longum, Bifidobacterium infantis, and Bifidobacterium breve
}

\author{
DRISS BAHAKA, ${ }^{1}$ CHRISTEL NEUT, ${ }^{2}$ ABDELKRIM KHATTABI, ${ }^{1}$ DANIEL MONGET, ${ }^{3}$ \\ AND FRANÇOISE GAVINI ${ }^{1,4 *}$
}

Institut National de la Recherche Agronomique, Laboratoire d'Ecologie et de Physiologie du Système Digestif, Domaine du C.E.R.T.I.A., 59650 Villeneuve d'Ascq Cedex, ${ }^{1}$ Service de Bacteriologie, Faculté de Pharmacie, 59045 Lille Cedex, ${ }^{2}$ Biomerieux-Unité de Bactériologie, La Balme les Grottes, 38390 Montalieu Vercieu, ${ }^{3}$ and Institut National de la Santé et de la Recherche Médicale, ${ }^{4}$ France

\begin{abstract}
A numerical analysis based on phenotypic characteristics (89 enzymatic tests and 49 carbohydrate acidification tests), in which experimental strips from Biomerieux-API, La Balme les Grottes, France, were used, was performed to characterize 82 new isolates belonging or related to Bifidobacterium longum, Bifidobacterium infantis, and Bifidobacterium breve. A total of 72 strains were isolated from child or adult feces, and the other strains were obtained from human vaginas and bronchi. In this study we also included 38 type and reference strains that were representative of all species of the genus Bifidobacterium and 6 strains belonging to the genus Lactobacillus. DNA-DNA relationships between $B$. longum and $B$. infantis were determined by using 19 strains related to these species, as determined by the numerical analysis. The degree of DNA binding was determined by the S1 nuclease method. The phenotypic study revealed that there were six main clusters, which were subdivided into nine subclusters. Subcluster Va contained the type strains of $B$. longum and $B$. infantis. The DNA-DNA relatedness values of some of the new isolates were very similar to the DNA-DNA relatedness values of the type strain of $B$. longum. On the basis of these data, it was difficult to isolate $B$. infantis strains and then to define $B$. infantis as a single species separated from $B$. longum. Subclusters IVb to IVf comprised reference strains of $B$. breve. Cluster III and subcluster Ia were not identified.
\end{abstract}

At the present time, the genus Bifidobacterium includes 29 species, 10 of which are of human origin. Four of these species, Bifidobacterium bifidum, Bifidobacterium longum, Bifidobacterium infantis, and Bifidobacterium breve, have been studied with increasing interest because of their role in physiological relationships in human (especially infant and child) gastrointestinal tracts $(2,3,29)$. Furthermore, today, the use of these bacteria in food microbiology (dairy products) makes accurate differentiation of these organisms necessary (19).

The type species of the genus, $B$. bifidum, is well separated from the other species on the basis of its phenotypic characteristics, as well as its DNA-DNA hybridization values. Identification of the three other species studied in this work is much more uncertain; in particular, distinction between $B$. longum and $B$. infantis is difficult.

On the basis of the results of phenotypic studies, Reuter (18) defined and subdivided $B$. longum into two biovars, biovars a and b, and Mitsuoka (17) divided $B$. longum into $B$. longum subsp. longum, and $B$. longum subsp. animalis variants $a$ and $b$. Variant a was elevated to species rank as Bifidobacterium animalis on the basis of the results of DNA-DNA hybridization experiments (22); however, phenotypic differentiation of this taxon from $B$. longum was still being discussed by Yaeshima et al. in 1991 (28).

The definitions of $B$. infantis and $B$. breve, as described by Reuter (18), were revised in 1971 by Scardovi et al. (24); $B$. infantis included Bifidobacterium liberorum and Bifidobacterium lactentis, species that were initially separated from $B$. infantis, while $B$. breve included Bifidobacterium parvulonum (18).

\footnotetext{
* Corresponding author.
}

The purpose of this study was to determine by numerical analysis and DNA-DNA hybridization the phenotypic and genomic characteristics of $B$. longum and $B$. infantis and the relationships of these species to $B$. breve.

\section{MATERIALS AND METHODS}

Bacterial strains. The strains which we used are listed in Table 1. A total of 126 strains were used in this study, including 82 new isolates (72 isolates from child and adult feces or intestines, 2 isolates from human vaginas, 2 isolates from bronchi, and 6 isolates of unknown origin) and 44 type and reference strains (6 of which belonged to the genus Lactobacillus). Every new isolate except strain R12H2 from an illeonal reservoir came from the Service de Bacteriologie, Faculté de Pharmacie de Lille, Lille, France.

Phenotypic characterization. All of the strains were considered members of the genus Bifidobacterium because they exhibited the characteristics described previously for this genus (10), especially the presence of fructose-6-phosphate phosphoketolase. The same tests performed previously (10), including 89 experimental enzymatic strip tests and 49 carbohydrate acidification tests (Biomerieux-API, La Balme les Grottes, France), were used for numerical analysis.

Numerical analysis. A total of 24 tests which were either positive or negative for all of the strains were not included in the numerical analysis. The levels of similarity between bacterial strains were calculated by using the Dice index (9). Aggregation was based on the unweighted pair group average-linkage method $(7,26)$.

G $+\mathbf{C}$ contents and DNA-DNA hybridization. DNA was extracted by using lysozyme (15) in addition to achromopeptidase treatment (1). After the lytic phase, purified DNA was obtained by the method of Marmur (15). The thermal dena- 
TABLE 1. Strains used in the numerical analysis

\begin{tabular}{|c|c|c|c|c|}
\hline Cluster & Subcluster & Strain $^{a}$ & Name as received & Isolated from: \\
\hline \multirow[t]{5}{*}{ I (5 strains) } & Ia & CUETM 89-129 & $?$ & Child feces \\
\hline & & CUETM 89-218 & $?$ & Child feces \\
\hline & & CUETM 89-244 & $?$ & Child feces \\
\hline & & CUETM 89-271 & $?$ & Child feces \\
\hline & Unclustered & $\begin{array}{l}\text { DSM 20097 } \\
(=\mathrm{C} 10-45)\end{array}$ & B. longum & Calf feces \\
\hline \multirow[t]{12}{*}{ II (11 strains) } & & DSM $20089^{\mathrm{T}}$ & B. asteroides & Honeybee \\
\hline & & $\begin{array}{l}\left(=\text { ATCC } 25910^{\mathrm{T}}=\mathrm{C}^{\mathrm{A}} 1^{\mathrm{T}}\right) \\
\text { DSM } 20214^{\mathrm{T}}\end{array}$ & B. indicum & Honeybee \\
\hline & & $\begin{array}{l}\left(=\text { ATCC } 25912^{\mathrm{T}}=\mathrm{C} 410^{\mathrm{T}}\right) \\
\text { DSM } 20434^{\mathrm{T}}\end{array}$ & B. choerinum & \\
\hline & & $\left(=\right.$ ATCC $27686^{\mathbf{T}}=$ SU806 $\left.^{\mathbf{T}}\right)$ & & Pig teces \\
\hline & & $\begin{array}{l}\operatorname{ATCC} 25865^{\mathrm{T}} \\
\left(=\mathrm{DSM} 20092^{\mathrm{T}}=\mathrm{RU} 224^{\mathrm{T}}\right)\end{array}$ & B. globosum & Bovine rumen \\
\hline & & $\begin{array}{l}\text { DSM 20099 } \\
\left(=\text { ATCC } 25526^{\mathrm{T}}=\text { PNC-2-9G }\right.\end{array}$ & B. pseudolongum & Pig feces \\
\hline & & $\begin{array}{l}\text { DSM } 20211^{\mathrm{T}} \\
\left(=\text { ATCC } 27533^{\mathrm{T}}=\mathrm{SU} 859^{\mathrm{T}}\right)\end{array}$ & B. suis & Pig feces \\
\hline & & $\begin{array}{l}\text { NCFB } 2242^{\mathrm{T}} \\
\left(=\text { ATCC } 25527^{\mathrm{T}}=\mathrm{R} 101-8^{\mathrm{T}}\right)\end{array}$ & B. animalis & Rat feces \\
\hline & & $\begin{array}{l}\text { DSM } 20222^{\mathrm{T}} \\
\left(=\text { ATCC } 27540^{\mathrm{T}}=\mathrm{RA}^{\mathrm{T}}\right)\end{array}$ & B. magnum & Rabbit feces \\
\hline & & DSM 20011 & L. casei subsp. casei & $?$ \\
\hline & & DSM 20079 & L. acidophilus & $?$ \\
\hline & & $\begin{array}{l}\text { DSM 20082 } \\
(=\mathrm{E} 319 \mathrm{f})\end{array}$ & B. bifidum & Adult feces \\
\hline \multirow[t]{3}{*}{ III (3 strains) } & IIIa & CUETM 89-158 & $?$ & Adult intestine \\
\hline & & CUETM 89-164 & $?$ & Child feces \\
\hline & Unclustered & $\begin{array}{l}\operatorname{DSM} 20096^{\mathrm{T}} \\
\left(=\text { ATCC } 27537^{\mathrm{T}}=\mathrm{F} 395^{\mathrm{T}}\right)\end{array}$ & B. subtile & Sewage \\
\hline \multirow{32}{*}{ IV (34 strains) } & & $\begin{array}{l}\text { DSM 20432 } \\
\left(=\text { ATCC } 27917^{\mathrm{T}}=\text { RU917 }\right.\end{array}$ & B. boum & Bovine rumen \\
\hline & IVb (11 strains) & CUETM 89-188 & $?$ & Child feces \\
\hline & & CUETM 89-205 & $?$ & Child feces \\
\hline & & CUETM 89-197 & $?$ & Child feces \\
\hline & & CUETM 89-185 & $?$ & Child feces \\
\hline & & CUETM 89-248 & $?$ & Child feces \\
\hline & & CUETM 89-156 & $?$ & $?$ \\
\hline & & CUETM 89-161 & $?$ & Child feces \\
\hline & & CUETM 89-155 & $?$ & $?$ \\
\hline & & CUETM 89-211 & $?$ & Child feces \\
\hline & & $\begin{array}{l}\text { ATCC } 15701 \\
(=\text { S46) }\end{array}$ & $\begin{array}{l}\text { B. breve } \\
\text { variant b }\end{array}$ & Child feces \\
\hline & & $\begin{array}{l}\text { DSM } 20091 \\
(=\text { ATCC } 15698=\text { S50) }\end{array}$ & B. breve & Child feces \\
\hline & IVc (6 strains) & CUETM 89-220 & $?$ & Child feces \\
\hline & & CUETM 89-154 & $?$ & Human vagina \\
\hline & & CUETM 89-243 & $?$ & Child feces \\
\hline & & CUETM 89-175 & $?$ & Human vagina \\
\hline & & CUETM 89-145 & $?$ & Child feces \\
\hline & & CUETM 89-149 & $?$ & Child feces \\
\hline & IVd (2 strains) & CUETM 89-160 & $?$ & Adult feces \\
\hline & & CUETM 89-183 & $?$ & Child feces \\
\hline & IVe (4 strains) & CUETM 89-182 & $?$ & Child feces \\
\hline & & CUETM 89-192 & ? & $?$ \\
\hline & & CUETM 89-190 & ? & $?$ \\
\hline & & CUETM 89-159 & $?$ & Child feces \\
\hline & IVf (4 strains) & CUETM 89-246 & $?$ & Child feces \\
\hline & & CUETM 89-272 & $?$ & Child feces \\
\hline & & CUETM 89-181 & $?$ & Child feces \\
\hline & & CUETM 89-163 & ? & Child feces \\
\hline & Unclustered & CUETM 89-184 & $?$ & Child feces \\
\hline & & CUETM 89-203 & $?$ & Child feces \\
\hline & & $\begin{array}{l}\text { NCFB } 2257^{\mathrm{T}} \\
\mathrm{T}\end{array}$ & B. breve & Infant intestine \\
\hline & & $\begin{array}{l}\left(=\text { DSM } 20213^{1}=\text { CCUG } 18365^{1}=S 1^{1}\right. \\
\text { CUETM 89-199 }\end{array}$ & $?$ & $?$ \\
\hline
\end{tabular}


TABLE 1-Continued

\begin{tabular}{|c|c|c|c|c|}
\hline Cluster & Subcluster & Strain $^{a}$ & Name as received & Isolated from: \\
\hline \multirow{53}{*}{$\mathrm{V}$ (52 strains) } & \multirow{39}{*}{ Va (38 strains) } & CUETM 89-327 & $?$ & Child feces \\
\hline & & CUETM 89-247 & ? & Child feces \\
\hline & & CUETM 89-287 & $?$ & Child feces \\
\hline & & CCUG 24071 & ? & Child feces \\
\hline & & CCUG 18157 & ? & $?$ \\
\hline & & CUETM 89-174 & ? & Child feces \\
\hline & & CUETM 89-186 & ? & Child feces \\
\hline & & CUETM 89-193 & $?$ & Child feces \\
\hline & & CUETM 89-263 & $?$ & Child feces \\
\hline & & CUETM 89-216 & $?$ & Adult intestine \\
\hline & & CUETM 89-215 & $?$ & Child feces \\
\hline & & CUETM 89-238 & $?$ & Child feces \\
\hline & & CUETM 89-281 & ? & Child feces \\
\hline & & CUETM 89-260 & $?$ & Child feces \\
\hline & & CUETM 89-257 & $?$ & Child feces \\
\hline & & CUETM 89-259 & $?$ & Child feces \\
\hline & & CUETM 89-267 & ? & Child feces \\
\hline & & CUETM 89-239 & $?$ & Child feces \\
\hline & & CUETM 89-276 & $?$ & Child feces \\
\hline & & CUETM 89-245 & $?$ & Child feces \\
\hline & & $\mathrm{R} 12 \mathrm{H} 2$ & ? & Pouchite \\
\hline & & C 76 & $?$ & Child feces \\
\hline & & CUETM 89-177 & $?$ & Child feces \\
\hline & & $\begin{array}{l}\text { NCTC } 11818^{\mathrm{T}} \\
\left(=\text { ATCC } 15707^{\mathrm{T}}=\mathrm{DSM} 20219^{\mathrm{T}}=\mathrm{E} 194 \mathrm{~b}^{\mathrm{T}}\right)\end{array}$ & B. longum & Adult feces \\
\hline & & CUETM 89-290 & $?$ & Child feces \\
\hline & & CCUG 15137 & ? & Adult intestine \\
\hline & & CUETM 89-172 & $?$ & $?$ \\
\hline & & CUETM 89-171 & ? & Child feces \\
\hline & & CUETM $89-268$ & $?$ & Child feces \\
\hline & & CUETM 89-265 & $?$ & Child feces \\
\hline & & CUETM 89-251 & $?$ & Child feces \\
\hline & & CUETM 89-256 & ? & Child feces \\
\hline & & CUETM 89-157 & ? & Child feces \\
\hline & & CUETM 89-284 & $?$ & Child feces \\
\hline & & CUETM 89-179 & $?$ & Child feces \\
\hline & & $\begin{array}{l}\text { DSM 20088 } \\
\left(=\text { ATCC } 15697^{\mathrm{T}}=S 12^{\mathrm{T}}\right)\end{array}$ & B. infantis & Child feces \\
\hline & & CUETM 89-170 & $?$ & Adult feces \\
\hline & & $\begin{array}{l}\text { ATCC } 15708 \\
(=\text { S3) }\end{array}$ & B. longum & Child feces \\
\hline & & $\begin{array}{l}\text { DSM } 20218 \\
(=\text { ATCC } 17930)\end{array}$ & B. infantis & $?$ \\
\hline & \multirow[t]{3}{*}{ Unclustered } & $\begin{array}{l}\operatorname{ATCC~} 33777^{\mathrm{T}} \\
\left(=\mathrm{CH} 206-5^{\mathrm{T}}\right)\end{array}$ & B. gallinarum & Chicken feces \\
\hline & & $\begin{array}{l}\text { ATCC } 15704 \\
(=\text { E305) }\end{array}$ & B. adolescentis & Adult feces \\
\hline & & CUETM 89-213 & $?$ & $?$ \\
\hline & \multirow{17}{*}{$\mathrm{Vb}$ (11 strains) } & CUETM $89-280$ & ? & Child feces \\
\hline & & CUETM 89-250 & $?$ & Child feces \\
\hline & & CUETM 89-262 & ? & Child feces \\
\hline & & CUETM 89-204 & $?$ & Child feces \\
\hline & & CUETM 89-128 & $?$ & Child feces \\
\hline & & CUETM 89-252 & ? & Child feces \\
\hline & & CUETM 89-167 & ? & Child feces \\
\hline & & CUETM 89-240 & ? & Child feces \\
\hline & & CUETM 89-253 & $?$ & Child feces \\
\hline & & CUETM 89-217 & $?$ & Child feces \\
\hline & & CUETM 89-269 & $?$ & Child feces \\
\hline \multirow{6}{*}{ VI (7 strains) } & & CUETM 89-236 & $?$ & Bronchus \\
\hline & & CUETM 89-285 & $?$ & Child feces \\
\hline & & $\begin{array}{l}\text { CCUG } 18363^{\mathrm{T}} \\
\left(=\mathrm{DSM} 20083^{\mathrm{T}}\right)\end{array}$ & B. adolescentis & Adult feces \\
\hline & & $\left.=\operatorname{ATCC} 15703^{T}=E 194 a^{T}\right)$ & & \\
\hline & & $\begin{array}{l}\text { CUETM 89-196 } \\
\text { DSM } 20438^{\mathrm{T}}\end{array}$ & $?$ & Child feces \\
\hline & & $\left(=\right.$ ATCC $\left.27919^{\mathrm{T}}=\mathrm{B} 1279^{\mathrm{T}}\right)$ & B. pseudocatenulatum & Child feces \\
\hline
\end{tabular}


TABLE 1-Continued

\begin{tabular}{|c|c|c|c|c|}
\hline Cluster & Subcluster & Strain $^{a}$ & Name as received & Isolated from: \\
\hline \multirow{15}{*}{ Unclustered } & & $\begin{array}{l}\text { CCUG } 18366^{\mathrm{T}}\left(=\operatorname{ATCC} 27539^{\mathrm{T}}=\text { DSM } 20103^{\mathrm{T}}\right. \\
\left.=\mathrm{B} 669^{\mathrm{T}}\right)\end{array}$ & B. catenulatum & Human feces \\
\hline & & $\begin{array}{l}\text { CCUG } 18367^{\mathrm{T}}\left(=\text { ATCC } 27534^{\mathrm{T}}=\text { DSM } 20436^{\mathrm{T}}\right. \\
\left.=\mathrm{B} 764^{\mathrm{T}}\right)\end{array}$ & B. dentium & Dental caries \\
\hline & & DSM $20216^{\mathrm{T}}\left(=\operatorname{ATCC} 25911^{\mathrm{T}}=\mathrm{C} 215^{\mathrm{T}}\right)$ & Bifidobacterium coryneforme & Honeybee \\
\hline & & DSM $20102^{\mathrm{T}}\left(=\operatorname{ATCC} 27538^{\mathrm{T}}=\mathrm{F} 392^{\mathrm{T}}\right)$ & Bifidobacterium minimum & Sewage \\
\hline & & DSM $20098^{\mathrm{T}}\left(=\operatorname{ATCC} 27535^{\mathrm{T}}=\mathrm{B} 677^{\mathrm{T}}\right)$ & Bifidobacterium angulatum & Adult feces \\
\hline & & DSM $20433^{\mathrm{T}}\left(=\operatorname{ATCC} 27685^{\mathrm{T}}=\mathrm{B} 145^{\mathrm{T}}\right)$ & Bifidobacterium pullorum & Chicken feces \\
\hline & & CUETM 89-209 & & Bronchus \\
\hline & & $\operatorname{DSM} 20016^{\mathrm{T}}\left(=\operatorname{ATCC} 23272^{\mathrm{T}}=\mathrm{F} 275^{\mathrm{T}}\right)$ & $\begin{array}{l}\text { Lactobacillus reuteri }(= \\
\text { Lactobacillus fermentum) }\end{array}$ & Adult intestine \\
\hline & & $\operatorname{DSM} 20074^{\mathrm{T}}\left(=\operatorname{ATCC} 9649^{\mathrm{T}}=\operatorname{NCIB} 8130^{\mathrm{T}}\right)$ & $\begin{array}{l}\text { Lactobacillus delbrueckii } \\
\text { subsp. delbrueckii }\end{array}$ & $?$ \\
\hline & & CUETM 89-166 & ? & Child feces \\
\hline & & CCUG 18650 & ? & \\
\hline & & $\operatorname{DSM} 20093^{\mathrm{T}}\left(=\mathrm{P}^{\mathrm{T}}\right)$ & Bifidobacterium gallicum & Human feces \\
\hline & & DSM $20435^{\mathrm{T}}\left(=\right.$ ATCC $\left.27916^{\mathrm{T}}=\mathrm{RA}^{\mathrm{T}} 93^{\mathrm{T}}\right)$ & Bifidobacterium cuniculi & Rabbit feces \\
\hline & & $\operatorname{DSM} 20505^{\mathrm{T}}\left(=\right.$ NCIB $\left.11720^{\mathrm{T}}\right)$ & Lactobacillus sharpae & Sewage \\
\hline & & CIP 3179 & Lactobacillus plantarum & \\
\hline
\end{tabular}

a ATCC, American Type Culture Collection, Rockville, Md.; C. Faculté de Pharmacie, Lille, France; CCUG, Culture Collection, University of Göteborg, Göteborg, Sweden; CIP, Collection Institut Pasteur, Paris, France; CUETM, Collection Unité Ecotoxicogie, Villeneuve d'Ascq, France; DSM, Deutsche Sammlung von Mikroorganismen, Göttingen, Germany; NCFB, National Collection of Food Bacteria, Shinfield, Reading, England; NCIB, National Collection of Industrial Bacteria, Aberdeen, Scotland; NCTC, National Collection of Type Cultures, Central Public Health Laboratory, London, United Kingdom; R. P. Raibaud, Laboratoire d'Ecologie et de Physiologie du Système Digestif, Institut National de la Recherche Agronomique, Jouy-en-Josas, France. upa ATCC, American Type Culture Collection, Rockville, Md.; C. Faculté de Pharmacie, Lille, France; CCUG, Culture Collection, University of Göteborg, ermentum)

turation and guanine-plus-cytosine $(\mathrm{G}+\mathrm{C})$ values were determined as described previously $(8,16)$. The degrees of DNA binding were determined by the $S 1$ nuclease-trichloroacetic acid method $(5,6,12)$, and the $\Delta T_{m}$ values $\left(\Delta T_{m}\right.$ is the difference between the temperature at which one-half of the reassociated DNA in the homologous reaction is denatured and the temperature at which one-half of the reassociated DNA in the heterologous reaction is denatured) were calculated $(5,12)$. The strains used in the DNA-DNA binding experiments and the results of these experiments are shown in Table 2.

\section{RESULTS}

Phenotypic characterization. Our dendrogram (Fig. 1) contained six main clusters (clusters I to VI), which were subdivided into nine subclusters (subclusters Ia, IVa to IVf, $\mathrm{Va}$, and $\mathrm{Vb}$ ). Fourteen strains did not fall into any cluster,

TABLE 2. $\mathrm{G}+\mathrm{C}$ contents of 19 strains belonging to subclusters $\mathrm{Va}$ and $\mathrm{Vb}$ and levels of DNA-DNA relatedness between these strains and $B$. longum and $B$. infantis type strains

\begin{tabular}{|c|c|c|c|c|c|c|}
\hline \multirow[b]{2}{*}{ Subcluster } & \multirow[b]{2}{*}{$\begin{array}{l}\text { No. of } \\
\text { strains }\end{array}$} & \multirow[b]{2}{*}{ Name as received } & \multirow[b]{2}{*}{ Strain } & \multirow[b]{2}{*}{$\underset{(\mathrm{mol} \%)}{\mathrm{G}+\mathrm{C} \text { content }}$} & \multicolumn{2}{|c|}{ \% DNA-DNA relatedness to: } \\
\hline & & & & & $\begin{array}{l}\text { B. longum } \\
\text { NCTC } 11818^{\mathrm{T}}\end{array}$ & $\begin{array}{l}\text { B. infantis } \\
\text { DSM } 20088^{\mathrm{T}}\end{array}$ \\
\hline \multirow[t]{15}{*}{$\mathrm{Va}$} & \multirow[t]{15}{*}{15} & B. longum & NCTC $11818^{T}$ & 57 & 100 & 66 \\
\hline & & Bifidobacterium sp. & CCUG 15137 & 57 & 96 & 65 \\
\hline & & Bifidobacterium $\mathrm{sp}$. & CUETM 89-239 & 59 & 89 & 68 \\
\hline & & Bifidobacterium sp. & CCUG 24071 & 57 & $89(2.0)^{a}$ & $60(5.5)$ \\
\hline & & Bifidobacterium sp. & CCUG 18157 & 57 & $86(3.0)$ & $64(5.6)$ \\
\hline & & Bifidobacterium $\mathrm{sp}$. & CUETM 89-260 & 57 & 86 & 62 \\
\hline & & Bifidobacterium sp. & CUETM $89-259$ & 56 & 84 & 67 \\
\hline & & B. longum & ATCC 15708 & 57 & 83 & 67 \\
\hline & & Bifidobacterium $\mathrm{sp}$. & CUETM 89-193 & 57 & $82(2.7)$ & $71(4.2)$ \\
\hline & & Bifidobacterium $\mathrm{sp}$. & CUETM 89-284 & 58 & $79(3.2)$ & $64(5.4)$ \\
\hline & & Bifidobacterium sp. & $\mathrm{R} 12 \mathrm{H} 2$ & 57 & $79(3.8)$ & $64(5.6)$ \\
\hline & & Bifidobacterium $\mathrm{sp}$. & CUETM 89-290 & 57 & 78 & 65 \\
\hline & & Bifidobacterium sp. & CUETM 89-170 & 57 & $76(2.5)$ & $61(5.9)$ \\
\hline & & B. infantis & DSM 20218 & 56 & $70(4.8)$ & $80(3.8)$ \\
\hline & & B. infantis & DSM $20088^{T}$ & 56 & $67(5.2)$ & 100 \\
\hline \multirow[t]{4}{*}{$\mathrm{Vb}$} & \multirow[t]{4}{*}{4} & Bifidobacterium sp. & CUETM 89-250 & 59 & $98(3.2)$ & $62(5.7)$ \\
\hline & & Bifidobacterium sp. & CUETM 89-252 & 57 & $95(2.7)$ & $62(5.4)$ \\
\hline & & Bifidobacterium sp. & CUETM 89-262 & 58 & $94(3.0)$ & $63(5.3)$ \\
\hline & & Bifidobacterium sp. & CUETM 89-240 & 57 & $77(2.2)$ & $70(4.9)$ \\
\hline
\end{tabular}

\footnotetext{
${ }^{a}$ The numbers in parentheses are $\Delta T_{m}$ values (in degrees Celsius).
} 


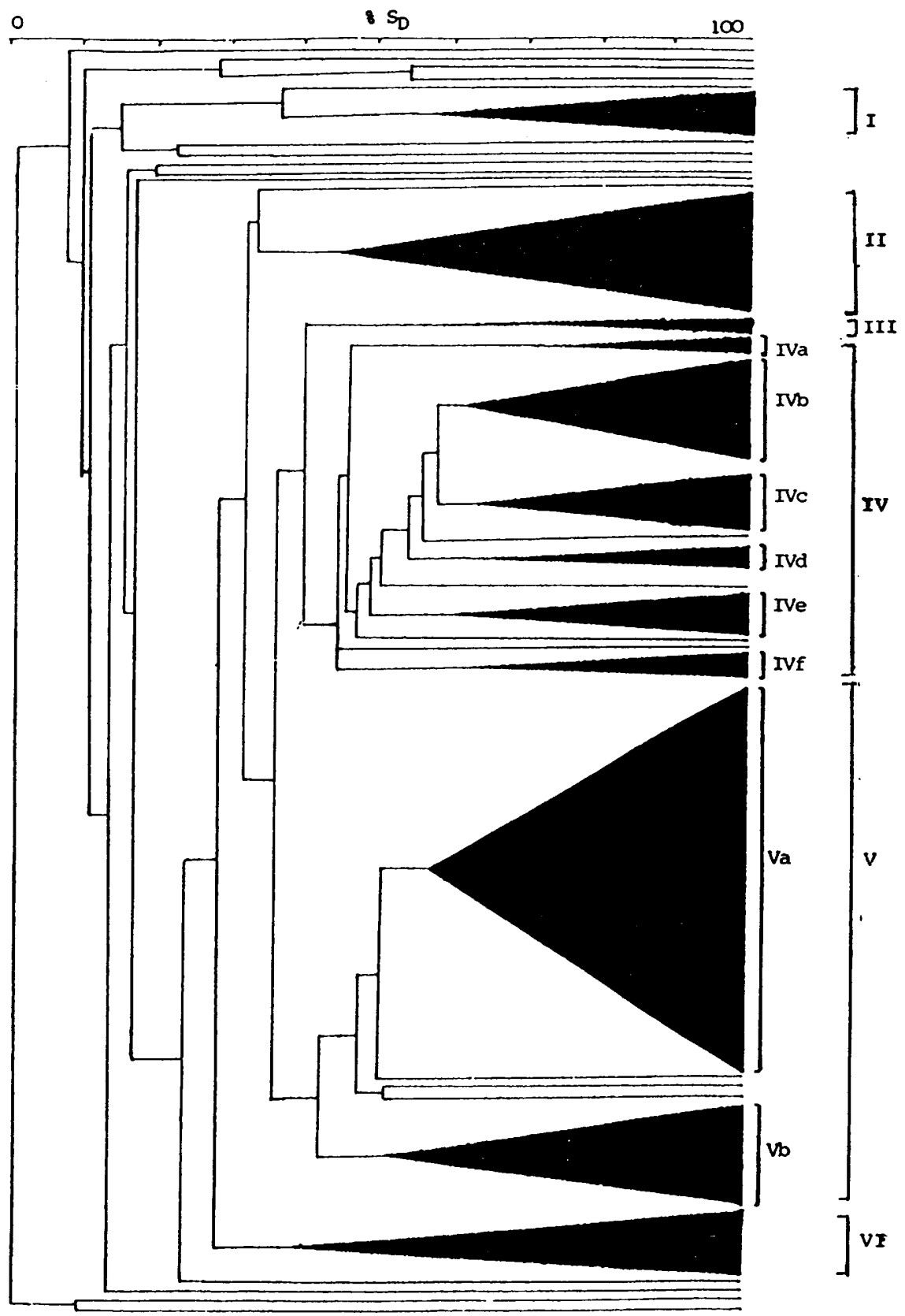

FIG. 1. Phenotypic dendrogram based on unweighted pair group average linkage.

and 10 strains were not included in any subcluster. The phenotypic characteristics of the clusters and subclusters are shown in Tables 3 and 4.

Cluster I. Cluster I comprised five strains, four of which were in subcluster Ia and were isolated from child feces. No type or reference strains were present in this cluster. Strain DSM 20097 (= C10-45), isolated from calf feces, was unclustered; its taxonomic position is discussed below.

Cluster II. Among the 11 strains in cluster II, 8 were type strains of Bifidobacterium species of animal origin, 2 were reference strains belonging to Lactobacillus casei and Lactobacillus acidophilus, and 1 was $B$. bifidum DSM 20082 (= E319f). Most of the type strains of animal origin and the
Lactobacillus strains were in one cluster. This artificial grouping could be easily explained by the phenotypic differentiation of these organisms from strains belonging to the other clusters.

Cluster III. The two strains in cluster III were isolated from an adult intestine and child feces. One strain, Bifido bacterium subtile DSM $20096^{\mathrm{T}}\left(=\mathrm{F} 395^{\mathrm{T}}\right)(\mathrm{T}=$ type strain $)$, which was isolated from sewage, was unclustered.

Cluster IV. The 34 strains in cluster IV were subdivided into six subclusters, and 5 strains were unclustered. Subcluster IVa contained the type strains of Bifidobacterium thermophilum (strain DSM 20210 [= P2-91]) and Bifidobacterium boum (strain DSM 20432 [= RU 917]), which were isolated 
TABLE 3. Characteristics for differentiating clusters I through $\mathrm{VI}^{a}$

\begin{tabular}{|c|c|c|c|c|c|c|}
\hline \multirow[b]{2}{*}{ Characteristic } & \multicolumn{6}{|c|}{$\%$ of strains positive } \\
\hline & $\begin{array}{l}\text { Cluster I } \\
(n=5)^{b}\end{array}$ & $\begin{array}{c}\text { Cluster II } \\
(n=11)\end{array}$ & $\begin{array}{c}\text { Cluster III } \\
(n=3)\end{array}$ & $\begin{array}{c}\text { Cluster IV } \\
(n=34)\end{array}$ & $\begin{array}{c}\text { Cluster V } \\
(n=52)\end{array}$ & $\begin{array}{l}\text { Cluster VI } \\
\quad(n=7)\end{array}$ \\
\hline \multicolumn{7}{|l|}{ Enzymatic tests } \\
\hline L-Tyrosine arylamidase & 0 & 100 & 100 & 41 & 41 & 9 \\
\hline L-Phenylalanine arylamidase & 0 & 100 & 100 & 97 & 90 & 18 \\
\hline L-Hydroxyproline arylamidase & 0 & 100 & 100 & 100 & 100 & 100 \\
\hline Glycine arylamidase & 0 & 82 & 0 & 68 & 83 & 9 \\
\hline L-Äsparate arylamidase & 89 & 100 & 0 & 85 & 100 & 18 \\
\hline$S$-benzyl-cysteine arylamidase & 0 & 100 & 67 & 62 & 65 & 0 \\
\hline Leucyl-glycine arylamidase & 0 & 91 & 0 & 62 & 71 & 0 \\
\hline L-Seryl-tyrosine arylamidase & 0 & 91 & 0 & 62 & 71 & 0 \\
\hline L-Proline arylamidase & 0 & 100 & 67 & 88 & 96 & 100 \\
\hline L-Histidyl-L-leucyl-L-histidine arylamidase & 40 & 100 & 0 & 88 & 96 & 27 \\
\hline L-Leucyl-L-alanine arylamidase & 0 & 100 & 0 & 18 & 56 & 0 \\
\hline L-Prolyl-L-arginine arylamidase & 0 & 45 & 0 & 3 & 85 & 18 \\
\hline L-Histidyl-L-phenylalanine arylamidase & 0 & 73 & 0 & 0 & 0 & 0 \\
\hline$\alpha$-D-Galactosidase & 0 & 100 & 100 & 97 & 85 & 55 \\
\hline$\alpha$-L-Arabinosidase & 100 & 64 & 0 & 0 & 96 & 100 \\
\hline$\alpha$-D-Glucosidase & 0 & 100 & 100 & 74 & 62 & 36 \\
\hline$\beta$-D-Glucosidase & 0 & 91 & 67 & 76 & 12 & 55 \\
\hline$\beta$-D-Fucosidase & 0 & 64 & 67 & 82 & 81 & 36 \\
\hline Esterase C4 & 0 & 100 & 100 & 59 & 79 & 36 \\
\hline Esterase C5 & 0 & 91 & 100 & 62 & 83 & 36 \\
\hline \multicolumn{7}{|l|}{ Carbohydrate acidification tests } \\
\hline L-Arabinose & 100 & 27 & 0 & 3 & 90 & 55 \\
\hline D-Xylose & 100 & 9 & 0 & 6 & 90 & 45 \\
\hline Melezitose & 80 & 0 & 0 & 6 & 65 & 0 \\
\hline Starch & 0 & 27 & 33 & 47 & 100 & 100 \\
\hline Amygdalin & 0 & 36 & 0 & 79 & 0 & 27 \\
\hline Mannitol & 0 & 18 & 67 & 82 & 2 & 0 \\
\hline Sorbitol & 0 & 9 & 100 & 82 & 4 & 27 \\
\hline
\end{tabular}

${ }^{a}$ The type strains of the following species were included in the clusters: cluster III, B. subtile; cluster IV, B. boum, B. thermophilum, and $B$. breve; cluster $\mathrm{V}, B$. longum and $B$. infantis; cluster VI, B. adolescentis, B. dentium, and $B$. catenulatum.

${ }^{b} n$ is the number of strains tested.

from pig feces and a bovine rumen, respectively. The other subclusters, subclusters IVb to IVf, contained only human strains. Subcluster IVb included two reference strains, $B$. breve ATCC 15701 (= S46) (variant b) and $B$. breve DSM 20091 (= ATCC $15698=$ S50) (variant a). The four other subclusters contained only new isolates. $B$. breve NCFB $2257^{\mathrm{T}}\left(=\mathrm{S} 1^{\mathrm{T}}\right)$ was unclustered, but not far from subcluster IVc.

Cluster V. Cluster $\mathrm{V}$ is the most important cluster (52 strains) and is divided into subcluster Va (38 strains) and subcluster $\mathrm{Vb}$ (11 strains). The following strains were not members of either of these subclusters: Bifidobacterium gallinarum ATCC $33777^{\mathrm{T}}\left(=\mathrm{CH} 206-5^{\mathrm{T}}\right)$, Bifidobacterium adolescentis ATCC 15704 (= E305), (variant b), and one strain whose origin was unknown.

The type strains of $B$. longum (strain NCTC 11818 [= E194b]) and $B$. infantis (strain DSM 20088 [= S12]) were in the same subcluster, subcluster $\mathrm{Va}$, along with two reference strains, $B$. longum ATCC $15708(=\mathrm{S} 3)$ (variant b) and $B$. infantis DSM 20218 (= ATCC 17930). Subcluster Vb did not contain any type or reference strains. All of these organisms were isolated from child feces.

Cluster VI. Cluster VI contained the type strains of species belonging to the $B$. adolescentis complex $(21,23)$, including $B$. adolescentis CCUG 18363 (= E194a), Bifidobacterium pseudocatenulatum DSM 20438 (= B1279), Bifidobacterium catenulatum CCUG 18366 (= B669), and Bifidobacterium dentium CCUG 18367 (= B764), and three wild strains, one isolated from a bronchus and two isolated from child feces. Thirteen strains were unclustered, including six type strains, four strains belonging to the genus Lactobacillus, and three new isolates (Table 1).

G $+C$ contents and DNA-DNA hybridization. The $\mathrm{G}+\mathrm{C}$ contents of 15 strains belonging to subcluster $\mathrm{Va}$ and 4 strains belonging to subcluster $\mathrm{Vb}$ ranged from 56 to 59 mol\% (Table 2).

The DNA relatedness values for these 19 strains, including the type strains of $B$. longum and $B$. infantis, are shown in Table 2. The levels of DNA-DNA base sequence similarity between these two type strains were 66 and $67 \%$. A higher value $(70 \%)$, with a $\Delta T_{m}$ of $4.8^{\circ} \mathrm{C}$, was obtained between $B$. infantis DSM 20218 and $B$. longum NCTC $11818^{\mathrm{T}}$ (= $\mathrm{E} 194 \mathrm{~b}^{\mathrm{T}}$ ), while a level of relatedness of $80 \%$ and a $\Delta T_{m}$ of $3.8^{\circ} \mathrm{C}$ were observed between $B$. infantis DSM 20218 and $B$. infantis DSM $20088^{\mathrm{T}}\left(=\mathrm{S} 12^{\mathrm{T}}\right)$.

The 11 new isolates belonging to subcluster $\mathrm{Va}$ and the 4 isolates belonging to subcluster $\mathrm{Vb}$ studied by DNA-DNA hybridization yielded levels of DNA relatedness with $B$. longum NCTC $11818^{\mathrm{T}}\left(=\mathrm{E} 194 \mathrm{~b}^{\mathrm{T}}\right)$ of more than $76 \%$. The reciprocal results obtained by using $B$. infantis DSM $20088^{\mathrm{T}}$ $\left(=\mathrm{S} 12^{\mathrm{T}}\right)$ as the reference strain ranged from 60 to $71 \%$, with $\Delta T_{m}$ values of less than $6.0^{\circ} \mathrm{C}$.

\section{DISCUSSION}

$B$. infantis and $B$. longum (subclusters $\mathrm{Va}$ and $\mathrm{Vb}$ ). $B$. infantis and $B$. longum strains, including the type strains, were not differentiated phenotypically, even on the basis of 
TABLE 4. Characteristics for differentiating subclusters IVa through IVf and subclusters Va and $\mathrm{Vb}^{a}$

\begin{tabular}{|c|c|c|c|c|c|c|c|c|}
\hline \multirow[b]{2}{*}{ Characteristic } & \multicolumn{8}{|c|}{$\%$ of strains positive } \\
\hline & $\begin{array}{c}\text { Subcluster } \\
\text { IVa } \\
(n=2)^{b}\end{array}$ & $\begin{array}{c}\text { Subcluster } \\
\text { IVb } \\
(n=11)\end{array}$ & $\begin{array}{c}\text { Subcluster } \\
\text { IVc } \\
(n=6)\end{array}$ & $\begin{array}{c}\text { Subcluster } \\
\text { IVd } \\
(n=2)\end{array}$ & $\begin{array}{c}\text { Subcluster } \\
\text { IVe } \\
(n=4)\end{array}$ & $\begin{array}{c}\text { Subcluster } \\
\text { IVf } \\
(n=4)\end{array}$ & $\begin{array}{c}\text { Subcluster } \\
\text { Va } \\
(n=38)\end{array}$ & $\begin{array}{c}\text { Subcluster } \\
\text { Vb } \\
(n=11)\end{array}$ \\
\hline \multicolumn{9}{|l|}{ Enzymatic tests } \\
\hline L-Phenylalanine arylamidase & 100 & 100 & 100 & 100 & 75 & 100 & 95 & 64 \\
\hline L-Alanine arylamidase & 0 & 100 & 100 & 50 & 50 & 100 & 95 & 91 \\
\hline$S$-benzyl-cysteine arylamidase & 100 & 91 & 83 & 0 & 0 & 25 & 79 & 27 \\
\hline Leucyl-glycine arylamidase & 50 & 82 & 100 & 50 & 0 & 25 & 92 & 0 \\
\hline L-Isoleucine arylamidase & 0 & 55 & 100 & 100 & 0 & 75 & 84 & 45 \\
\hline L-Threonine arylamidase & 0 & 91 & 100 & 100 & 0 & 100 & 63 & 45 \\
\hline $\begin{array}{l}\text { L-Alanyl-L-phenylalanyl-L- } \\
\text { proline arylamidase }\end{array}$ & 100 & 100 & 67 & 0 & 50 & 25 & 66 & 0 \\
\hline $\begin{array}{l}\text { L-Histidyl-L-serine } \\
\text { arylamidase }\end{array}$ & 0 & 9 & 17 & 100 & 0 & 25 & 71 & 9 \\
\hline L-Lysyl-L-alanine arylamidase & 0 & 45 & 83 & 100 & 0 & 75 & 58 & 9 \\
\hline$\alpha$-D-Galactosidase & 100 & 100 & 100 & 100 & 100 & 75 & 97 & 18 \\
\hline$\alpha$-D-Glucosidase & 100 & 91 & 67 & 50 & 100 & 0 & 74 & 9 \\
\hline$\beta$-D-Fucosidase & 50 & 100 & 100 & 100 & 100 & 0 & 92 & 27 \\
\hline$\beta$-D-Lactosidase & 0 & 64 & 100 & 50 & 0 & 0 & 5 & 9 \\
\hline$\beta$-D-Xylosidase & 0 & 18 & 100 & 50 & 0 & 0 & 0 & 0 \\
\hline Esterase C4 & 100 & 75 & 67 & 100 & 25 & 0 & 97 & 0 \\
\hline Esterase C5 & 100 & 75 & 33 & 100 & 75 & 0 & 100 & 0 \\
\hline Esterase $\mathrm{C} 10$ & 100 & 82 & 33 & 50 & 25 & 0 & 18 & 0 \\
\hline \multicolumn{9}{|l|}{ Carbohydrate acidification tests } \\
\hline L-Arabinose & 0 & 0 & 0 & 0 & 0 & 0 & 92 & 100 \\
\hline Ribose & 0 & 82 & 67 & 100 & 100 & 100 & 61 & 64 \\
\hline D-Xylose & 0 & 0 & 0 & 0 & 0 & 0 & 89 & 100 \\
\hline Cellobiose & 0 & 9 & 50 & 50 & 100 & 0 & 3 & 0 \\
\hline Trehalose & 0 & 50 & 0 & 0 & 0 & 0 & 0 & 0 \\
\hline Melezitose & 0 & 0 & 17 & 0 & 0 & 0 & 68 & 55 \\
\hline Mannitol & 0 & 91 & 100 & 0 & 100 & 100 & 3 & 0 \\
\hline Sorbitol & 0 & 91 & 100 & 0 & 100 & 100 & 5 & 0 \\
\hline Starch & 50 & 9 & 83 & 100 & 50 & 25 & 3 & 0 \\
\hline
\end{tabular}

${ }^{a}$ The type strains and reference strains of the following species were included in the subclusters: subcluster IVa, B. thermophilum and B. boum; subcluster $\mathrm{IVb}, B$. breve variants a and $\mathrm{b}$; subcluster $\mathrm{Va}, B$. longum and $B$. infantis.

${ }^{b} n$ is the number of strains tested.

a large number of tests (carbohydrate acidification and enzymatic tests). Scardovi et al. (20) referred to the sugar fermentation patterns extensively shared by these two species. L-Arabinose and D-xylose, which are not fermented by strains identified as $B$. infantis according to Reuter (18), were acidified by most of the $B$. infantis strains studied by Scardovi et al. (20). All strains were melezitose negative according to both studies. Melezitose and D-ribose reactions could be positive or negative with members of the $B$. longum group according to Scardovi et al. (20), while melezitose and L-arabinose reactions were nearly always positive for $B$. longum strains according to Biavati et al. (4). These authors observed overlapping of zymograms (transaldolases and 6-phosphogluconate dehydrogenases) for the two species. In 1991, Roy et al. (19) described, on the basis of a few reference strains, specific biochemical tests for the differentiation of Bifidobacterium species. L-Arabinose acidification and melezitose acidification were used by these authors to separate $B$. infantis and $B$. longum after a numerical analysis based on carbohydrate acidification tests in which API Rapid $\mathrm{CH}$ galleries obtained from Analytab Products were used. The absence of new isolates made it impossible to conclude that these tests were sufficient to separate the two species.

Strains in subclusters $\mathrm{Va}$ and $\mathrm{Vb}$ were L-arabinose and D-xylose positive, as reported by Scardovi et al. (20) for their $B$. infantis strains. However, 61 and $64 \%$ of the strains in subclusters $\mathrm{Va}$ and $\mathrm{Vb}$ were ribose positive, respectively, and 68 and $55 \%$ of the strains were melezitose positive, respectively. These results are consistent with the results described by Scardovi et al. (20) for B. longum strains, but not with the results of Biavati et al. (4), who found that $92 \%$ of their $B$. longum strains were melezitose positive. Therefore, these characteristics could not be used either to differentiate the two species or to identify subclusters $\mathrm{Va}$ and $\mathrm{Vb}$ as members of one of these species (Table 4). Differentiation between the two subclusters was based on enzymatic tests, and the data indicated that there are two different phenotypes for the $B$. longum-B. infantis group.

The levels of DNA relatedness between the two species were found to vary from 65 to $80 \%(20,23,25)$. Later, in 1983, Lauer and Kandler (14) performed DNA-DNA hybridization experiments with the type strains and obtained values of 62 to $63 \%$. Furthermore, DNAs from strains isolated from calves were as closely related ( 75 to $80 \%)$ to $B$. infantis strains as to $B$. longum strains and thus could represent part of a "continuum" between the two species (20).

Our results for 19 human strains (Table 2) showed that their levels of DNA-DNA relatedness were 67 to 98 and 61 to $80 \%$ when $B$. longum and $B$. infantis, respectively, were used as the reference strains. Of the $12 \Delta T_{m}$ values calculated (Table 2), 11 were less than $5^{\circ} \mathrm{C}$ in the experiments performed with $B$. longum as the reference strain; the $\Delta T_{m}$ for the type strain of $B$. infantis was $5.2^{\circ} \mathrm{C}$. In contrast, eight 
$\Delta T_{m}$ values were more than $5^{\circ} \mathrm{C}$ in the experiments performed with $B$. infantis as the reference strain, but three values were less than $5^{\circ} \mathrm{C}$. According to the definition of a species, as cited by Wayne et al. (27), and the standard errors calculated by Grimont et al. (13) (about $4 \%$ in the S1 nuclease method and about $1.7^{\circ} \mathrm{C}$ in $\Delta T_{m}$ determinations), only one strain (DSM 20218) could be considered a $B$. infantis strain. Two new isolates (CUETM 89-193 and CUETM 89-240) could represent part of the continuum described by Scardovi et al. (20). All of the other strains are B. longum strains.

$B$. breve (subclusters IVb to IVf). Although the type strain of $B$. breve, strain NCFB 2257, was unclustered in subclusters IVb to IVf, the five subclusters could be assigned to the species $B$. breve on the basis of acidification of carbohydrates according to Scardovi et al. (24) and Biavati et al. (4). The acidification of mannitol and the acidification of sorbitol (except for the two strains in subcluster IVd) are particularly important. All of the strains were L-arabinose or D-xylose negative. In subclusters IVb to IVf $33 \%$ of the strains were cellobiose negative. Subcluster IVb included two reference strains, $B$. breve ATCC 15701 (= S46) and DSM 20091 (= ATCC $15698=$ S50). Strain ATCC 15698 was not grouped, according to Roy et al. (19), with the other two $B$. breve strains tested by these authors (strains ATCC $15700[=\mathrm{S} 1]$ and ATCC 15701 [= S46]). It is difficult to differentiate a species on the basis of only three strains, particularly when one strain has atypical characteristics. Enzymatic tests were useful in this study in view of the separation of the five subclusters (Table 4).

The DNA-DNA relatedness values for most of the strains phenotypically identified as $B$. breve when type strain $\mathrm{S} 1$ was used as the reference strain were very similar (between 75 and $91 \%$ ) (24).

Cluster VI. Cluster VI contained four type strains, $B$. adolescentis CCUG 18363 (= E194a), B. pseudocatenulatum DSM 20438 (= B1279), B. catenulatum CCUG 18366 (= B669), and B. dentium CCUG 18367 (= B764), and three new isolates. These genospecies were defined by Scardovi et al. $(21,23)$ on the basis of the phenospecies described by Reuter (18). These species were previously placed in the $B$. adolescentis homology group (21) and the B. pseudocatenulatum group (23).

Unidentified clusters. (i) Cluster I. It was interesting that one of the strains (CUETM 89-271) isolated from child feces was also studied by Gavini et al. (10) and clustered in their group VIIa but was not assigned to any known species. In this study, strain DSM $20097(=\mathrm{C} 10-45)$ was unclustered in cluster I. It was received from the Deutsche Sammlung von Mikroorganismen as $B$. longum because of its high DNADNA relatedness to the $B$. longum type strain (89\%) according to Lauer and Kandler (14). In 1971, Scardovi et al. (24) defined this strain as $B$. longum subsp. animalis biotype $b$, as Mitsuoka did (17), but established that it was $100 \%$ homologous with Bifidobacterium pseudolongum biotype c strain $29 \mathrm{SrT}$ and $65 \%$ homologous with $B$. pseudolongum biotype a strain PNC-2-9G, which is the type strain of the species at the present time. Its taxonomic position in our study did not permit us to determine to which species it belonged. The discrepancies between the results of Scardovi et al. (24) and those of Lauer and Kandler (14) might be explained by differences in the strains used since they were obtained from different collections and were studied by different authors.

(ii) Cluster III. Cluster III comprised subcluster IIIa, with only two strains, and the type strain of $B$. subtile, strain DSM 20096. One of the two strains, CUETM 89-158, was clustered by Gavini et al. (10) with the $B$. catenulatum type strain (CCUG 18366). In our analysis, this type strain was included in cluster VI, along with other type strains belonging to the $B$. adolescentis complex. This difference in taxonomic position could be explained by the small number of strains belonging to this complex which were studied.

In conclusion, we make the following observations. $B$. longum and $B$. infantis could not be differentiated on the basis of a large number of phenotypic characteristics. The genomic data obtained in this study lead us to ask whether the existence of strains named $B$. infantis is a reality, since no new strains have been isolated.

Type strain $B$. infantis DSM 20088 and reference strain $B$. infantis DSM 20218 could be considered marginal strains belonging to the species $B$. longum. $B$. breve has been divided into five phenospecies; however, this finding probably is not supported by genomic data if we consider previous DNA-DNA hybridization results for different phenotypes. The strains of human origin in cluster I and subcluster IIIa, which phenotypically are related to $B$. longum, $B$. infantis, and $B$. breve, could not be identified as members of any known species. Further DNA-DNA hybridization studies must be carried out to define the taxonomic position of these organisms.

\section{ACKNOWLEDGMENT}

We thank G. Bachelet for technical assistance.

\section{REFERENCES}

1. Barsotti, O., J. J. Morrier, J. Freney, F. Renaud, G. Benay, D. Decoret, and J. Dumont. 1988. Achromopeptidase for rapid lysis of oral anaerobic Gram-positive rods. Oral Microbiol. Immunol. 3:86-88.

2. Bezkorovainy, A. 1989. Ecology of bifidobacteria. In A. Bezkorovainy and R. Miller-Catchpole (ed.), Biochemistry and physiology of bifidobacteria. CRC Press, Boca Raton, Fla.

3. Biavati, B., P. Castagnoli, F. Crociani, and L. D. Trovatelli. 1984. Species of Bifidobacterium in the feces of infants. Microbiologica (Bologna) 7:341-345.

4. Biavati, B., V. Scardovi, and W. E. C. Moore. 1982. Electrophoretic patterns of proteins in the genus Bifidobacterium and proposal of four new species. Int. J. Syst. Bacteriol. 32:358-373.

5. Brenner, D. J. 1978. Characterization and clinical identification of Enterobacteriaceae by DNA hybridization. Prog. Clin. Pathol. 7:71-117.

6. Crosa, J. M., D. J. Brenner, and S. Falkow. 1973. Use of a single-strand-specific nuclease for analysis of bacterial and plasmid deoxyribonucleic acid homo- and heteroduplexes. J. Bacteriol. 115:904-911.

7. Delabre, M., A. Bianchi, and M. Veron. 1973. Etude critique des méthodes de taxonomie numérique. Application à une classification des bactéries aquicoles. Ann. Inst. Pasteur Microbiol. 124A:489-506.

8. De Ley, J. 1970. Reexamination of the association between melting point, buoyant density, and chemical base composition of deoxyribonucleic acid. J. Bacteriol. 101:738-754.

9. Gavini, F., B. Lefebvre, and H. Leclerc. 1976. Positions taxonomiques d'entérobactéries H2S- par rapport au genre Citrobacter. Ann. Inst. Pasteur Microbiol. 127A:275-295.

10. Gavini, F., A. M. Pourcher, C. Neut, D. Monget, C. Romond, C. Oger, and D. Izard. 1991. Phenotypic differentiation of bifidobacteria of human and animal origins. Int. J. Syst. Bacteriol. 41:548-557.

11. Gavini, F., M. B. Romond, A. Beji, C. Bernard, and D. Izard. 1990. Genomic and phenotypic characterizations of Pseudomonas alcaligenes and Comamonas species with a special reference to C. testosteroni. Curr. Microbiol. 21:279-282.

12. Grimont, P. A. D. 1988. Use of DNA reassociation in bacterial classification. Can. J. Microbiol. 34:541-546. 
13. Grimont, P. A. D., M. Y. Popoff, F. Grimont, C. Coynault, and M. Lemelin. 1980. Reproductibility and correlation study of three deoxyribonucleic acid hybridization procedures. Curr. Microbiol. 4:325-330.

14. Lauer, E., and O. Kandler. 1983. DNA-DNA homology, murein types, and enzyme patterns in the type strains of the genus Bifidobacterium. Syst. Appl. Microbiol. 4:42-64.

15. Marmur, J. 1961. A procedure for isolation of deoxyribonucleic acid from microorganisms. J. Mol. Biol. 3:208-218.

16. Marmur, J., and P. Doty. 1962. Determination of base composition of deoxyribonucleic acid from its thermal denaturation temperature. J. Mol. Biol. 5:109-118.

17. Mitsuoka, T. 1969. Vergleichende Untersuchungen über die Bifidobakterien aus dem Verdauungstrakt von Menschen und Tieren. Zentralbl. Bakteriol. Parasitenkd. Infektionskr. Hyg. Abt. 1 Orig. 210:52-64.

18. Reuter, G. 1963. Vergleichende Untersuchungen über die Bifidus-Flora im Säuglings- und Erwachsenenstuhl. Zentralbl. Bakteriol. Parasitenkd. Infektionskr. Hyg. Abt. 1 Orig. 191:486507.

19. Roy, D., P. Ward, C. J. Toupin, and P. Chevalier. 1991. Phenotypic characterization of Bifidobacterium spp. of human origin using rapid method. Microbiol. Aliments Nutr. 9:133-138.

20. Scardovi, V., F. Casalicchio, and N. Vincenzi. 1979. Multiple electrophoretic forms of transaldolase and 6-phosphogluconic dehydrogenase and their relationships to the taxonomy and ecology of the bifidobacteria. Int. J. Syst. Bacteriol. 29:312-327.

21. Scardovi, V., and F. Crociani. 1974. Bifidobacterium catenulatum, Bifidobacterium dentium, and Bifidobacterium angulatum: three new species and their deoxyribonucleic acid homology relationships. Int. J. Syst. Bacteriol. 24:6-20.
22. Scardovi, V., and L. D. Trovatelli. 1974. Bifidobacterium animalis (Mitsuoka) comb. nov. and the "minimum" and "subtile" groups of new bifidobacteria found in sewage. Int. J. Syst. Bacteriol. 24:21-28.

23. Scardovi, V., L. D. Trovatelli, B. Biavati, and G. Zani. 1979. Bifidobacterium cuniculi, Bifidobacterium choerinum, Bifidobacterium boum, and Bifidobacterium pseudocatenulatum: four new species and their deoxyribonucleic acid homology relationships. Int. J. Syst. Bacteriol. 29:291-311.

24. Scardovi, V., L. D. Trovatelli, G. Zani, F. Crocciani, and D. Mateuzzi. 1971. Deoxyribonucleic acid homology relationships among species of the genus Bifidobacterium. Int. J. Syst. Bacteriol. 21:276-294.

25. Scardovi, V., G. Zani, and D. Trovatelli. 1970. Deoxyribonucleic acid homology among the species of the genus Bifidobacterium isolated from animals. Arch. Microbiol. 72:318-325.

26. Sneath, P. H. A., and R. R. Sokal. 1973. Numerical taxonomy. W. H. Freeman, San Francisco.

27. Wayne, L. G., D. J. Brenner, R. R. Colwell, P. A. D. Grimont, O. Kandler, M. I. Krichevsky, L. H. Moore, W. E. C. Moore, R. G. E. Murray, E. Stackebrandt, M. P. Starr, and H. G. Trüper. 1987. Report of the Ad Hoc Committee on Reconciliation of Approaches to Bacterial Systematics. Int. J. Syst. Bacteriol. 37:463-464.

28. Yaeshima, T., T. Fujisawa, and T. Mitsuoka. 1991. Differential characteristics of Bifidobacterium longum and Bifidobacterium animalis. Syst. Appl. Microbiol. 14:169-172.

29. Yamazaki, S., K. Machii, S. Tsuyuki, H. Momose, T. Kawashima, and K. Ueda. 1985. Immunological responses to monoassociated Bifidobacterium longum and their relation to prevention of bacterial invasion. Immunology 56:43-50. 